\title{
Profile of anti-Leishmania antibodies related to clinical picture in canine visceral leishmaniasis
}

\author{
José Cláudio Carneiro de Freitas ${ }^{\mathrm{a}, *}$, Belarmino Eugênio Lopes-Neto ${ }^{\mathrm{a}}$, Cyntia Rafaelle Amaral de Abreu ${ }^{\mathrm{a}}$, \\ Wendel Coura-Vital $^{\text {b }}$, Samuel Leôncio Braga ${ }^{\text {b }}$, Alexandre Barbosa Reis ${ }^{b}$, \\ Diana Célia Sousa Nunes-Pinheiro ${ }^{\mathrm{a}}$

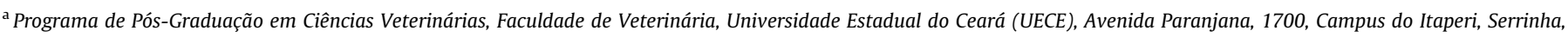 \\ CEP 60740-000, Fortaleza, CE, Brazil

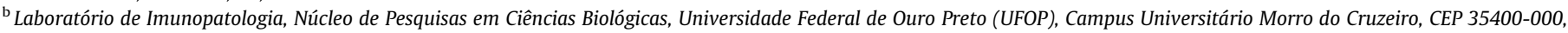 \\ Ouro Preto, MG, Brazil
}

\section{A R T I C L E I N F O}

Article history:

Received 19 July 2011

Accepted 5 December 2011

\section{Keywords:}

Canine visceral leishmaniasis

Anti-Leishmania antibodies

Clinical picture

Leishmania chagasi

\begin{abstract}
A B S T R A C T
This research investigated the profile of anti-Leishmania antibodies in different clinical forms of canine visceral leishmaniasis (CVL). Naturally infected dogs were divided into two groups: subclinical dogs (SD, $n=10)$ and clinical dogs (CD, $n=68)$. Non-infected dogs (ND, $n=7)$ comprised the negative control group. The humoral response was evaluated by the profile of total IgG, IgG1, IgG2, IgM, IgA and IgE, determined by ELISA. Infected animals showed increased levels of total IgG, IgA and IgE in addition to IgG1 and IgG2 in groups SD and CD, when compared with group ND. Furthermore, it was observed that IgG2 and IgM were correlated with symptomatology, while total IgG, IgG1 and IgA were negatively correlated and IgE showed no correlation. It follows that serum levels of IgG2 anti-Leishmania are correlated with typical clinical signs of disease. Furthermore the determination of specific anti-Leishmania antibodies could be an important tool in monitoring CVL clinical picture.
\end{abstract}

Crown Copyright @ 2011 Published by Elsevier Ltd. All rights reserved.

\section{Introduction}

The leishmaniasis are a complex of infectious diseases caused by different species of protozoa of the genus Leishmania. The infection is transmitted by the bite of infected insects of the genus Phlebotomus and Lutzomyia in the New and Old World, respectively (Peters and Sacks, 2006). In Brazil, the Programme for the Control of Visceral Leishmaniasis recommends early diagnosis and treatment of human cases, vector control and the detection and euthanasia of seropositive dogs (Brasil, 2006).

Although humans can also act as reservoir of the agent and plays a role in the transmission cycle, the dog is considered one of the most important links in the epidemiological chain of leishmaniasis (Ribeiro, 2007). Studies conducted in São Paulo showed a prevalence of visceral leishmaniasis up to $40 \%$ in the canine population (Ikeda et al., 2003). However, the prevalence in endemic areas may reach higher levels, as demonstrated by Freitas et al. (2010), through an epidemiological survey in Fortaleza, whose highest and lowest prevalence was $80.2 \%$ and $64 \%$ respectively.

Dogs with clinical leishmaniasis (CD) are characterized by clinical signs and/or clinical pathological abnormalities, with infection

\footnotetext{
* Corresponding author. Tel.: +55 8531019859 .

E-mail address: joseclaudiocarneiro@yahoo.com.br (J.C.C. de Freitas).
}

confirmed by specific tests, while dogs with subclinical leishmaniasis (SD) are characterized by no clinical signs or clinicopathological abnormalities, but with confirmed infection (Solano-Gallego et al., 2009).

The onset of clinical signs in canine visceral leishmaniasis (CVL) involves a number of factors, and these are associated with the animal's immune response (Ciaramella and Corona, 2003). In (CVL), the immune response mediated by Th1 lymphocytes, that secrete stimulatory cytokines (IFN- $\gamma$ ), activates macrophages infected, effectively controlling the infection (Miranda et al., 2007). On the other hand, when the immune response is mediated by Th2 lymphocytes, IL-4 secretors, there is a high production of antibodies that are associated with severe clinical manifestations (Miranda et al., 2007). It is noteworthy that when the immune response is mediated by regulatory $\mathrm{T}$ lymphocytes, producing IL-10, Th1 is inhibited and the infection is worsen (Miyara and Sakaguchi, 2007; Belkaid and Tarbell, 2009).

Studies report the role of antibodies in the CVL, relating the clinical picture with the presence of different classes and subclasses of immunoglobulins involved in the inflammatory response (TrotzWilliams and Gradoni, 2003; Almeida et al., 2005). The soluble immune complexes are deposited in various organs and tissues such as kidneys, blood vessels, joints, among others, favoring the appearance of various symptoms such as epistaxis, polyuria and 
polydipsia, uveitis, conjunctivitis and episcleritis immune-mediated, skin ulcers and tips of ears, hyperkeratosis and limping by poliartritre (Ciaramella and Corona, 2003).

Given the great importance of the disease, this study aims to evaluate the profile of anti-Leishmania antibodies in different clinical pictures of CVL with emphasis on correlation with the clinical symptoms.

\section{Material and methods}

\subsection{Animals}

Adult dogs $(n=85)$ were used, varying in age, weight and breeds (including cross-breed). The seropositive dogs were captured by the Zoonosis Control Center of Fortaleza (CCZ), as a CVL control measure. This study was approved by the Ethics Committee for Animal Use of the State University of Ceará (CEUA/UECE), protocol number 08622833-1.

\subsection{Immunofluorescence assay (IFA)}

In all animals the immunofluorescence assay (IFA) was performed for canine visceral leishmaniasis, being considered seropositive dogs the ones with titers above of $1: 40$, according to recommendations of the Ministry of Health of Brazil. Serologic testing was performed at CCZ using Bio-Manguinhos (FIOCRUZ$\mathrm{RJ})$ kits, following the manufacturer's recommendations.

\subsection{Parasitological diagnosis}

After anesthetizing the dog with Xylazine $(2 \mathrm{mg} / \mathrm{kg}$ ) and Ketamine $(10 \mathrm{mg} / \mathrm{kg})$ bone marrow aspiration for making imprints on microscope slides was performed. This material was fixed with methanol and stained with Panótipo fast dye. The stained imprints were observed under an optical microscope, and samples where the presence of amastigotes of Leishmania chagasi as revealed were considered positive

\subsection{Clinical classification}

All dogs were examined by observing the typical clinical signs of canine visceral leishmaniasis as onychogryphosis, hepatosplenomegaly, cachexia, lymphadenopathy, keratoconjunctivitis, skin ulcers, apathy, alopecia.

The dogs were divided into three groups, according to SolanoGallego et al. (2009). Negative dogs (ND =7), which do not show clinical and laboratory alterations (hematology and biochemistry) and negative for visceral leishmaniasis, by serology and parasitology; subclinical dogs $(S D=10)$ which do not show clinical and laboratory alterations and positive for Leishmania chagasi infection or as clinical dogs $(C D=68)$ which show clinical and laboratory alterations for routine testing and have infection confirmed by serological and parasitological diagnosis.

\subsection{Collection of blood samples}

Blood samples were collected by jugular venipuncture with a sterile syringe on dogs of different groups which were placed into a tube containing gel separation, without anticoagulant to obtain serum. Sera samples were stored at $-20^{\circ} \mathrm{C}$ for further test.

\subsection{Immunoenzymatic reaction - ELISA}

To determine the profile of anti-Leishmania antibodies, ELISA assay was performed, using soluble antigen (MHOM/BR/1972/BH46) from promastigotes of $L$. chagasi (SLA) from the axenic culture in LIT medium (Reis et al., 2006).

96-well microplates (Maxisorp ${ }^{\mathrm{TM}}$ Nunc International Nalgas, USA) were coated with SLA at a concentration of $2 \mathrm{mg} /$ well, overnight at $4{ }^{\circ} \mathrm{C}$. After incubation, the plates were washed four times with phosphate buffer solution (PBS) containing $0.05 \%$ Tween 20 and blocked for $45 \mathrm{~min}$ at $37^{\circ} \mathrm{C}$ with $100 \mathrm{~mL}$ of fetal bovine serum (5\%) in PBS per cell. Then, serum samples were added at a dilution of 1:80 for $\operatorname{IgG}, \operatorname{IgG} 1, \operatorname{IgG} 2$, IgM and $\operatorname{IgE}$ and 1:40 for IgA. After this procedure, they were washed and added peroxidase conjugate (Bethyl Laboratories Inc., Montgomery, TX, USA) previously diluted as follows: anti-dog IgG1 (anti-heavy chain specific), 1:8000; IgM (anti-m chain specific), 1:1000; IgA (anti-a chain specific), 1:500; IgE (anti-e chain specific), 1:500, or anti-dog IgG and IgG2 (both anti-heavy chain specific), 1:16.000. After four washings (as described above), the reaction was started by adding $100 \mathrm{~mL}$ of $0.1 \mathrm{M}$ citrate solution ( $\mathrm{pH} 5.0$ ) containing $0.03 \% \alpha$-phenylenediamine and $0.012 \% \mathrm{H}_{2} \mathrm{O}_{2}$, followed by incubation at $37{ }^{\circ} \mathrm{C}$ for $10 \mathrm{~min}$. The reaction was stopped by adding $32 \mu \mathrm{L}$ of $\mathrm{H}_{2} \mathrm{SO}_{4}$ $2.5 \mathrm{M}$ and the absorbance $(492 \mathrm{~nm})$ was measured with a plate reader-ELISA ELX800 (Biotek Instruments ${ }^{\circledR}$ VT, USA).

\subsection{Statistical analysis}

Statistical analysis was performed using the software GraphPad Prism 5.0. To compare the absorbance values of anti-Leishmania antibodies among groups in different clinical forms, analysis of variance (ANOVA) one-way was used, followed by Tukey test. To identify the association between the profile of anti-Leishmania antibodies and the clinical symptoms, we used the Spearman correlation test $(r)$. In all cases the differences were considered significant at $P \leqslant 0.05$.

\section{Results}

The results of the evaluation of typical clinical signs of CVL were expressed in percentage (\%) and are shown in Table 1 . The more frequent clinical signs were cachexia (77.94\%), keratoconjunctivitis (61.76\%) and lymphadenopathy (55.88\%), and $86.76 \%$ of the animals showed more than one typical clinical sign of CVL.

The profile of anti-Leishmania serum antibodies (total IgG, IgG1, $\operatorname{IgG}$, IgM, IgA and IgE) from naturally infected dogs in relation to different clinical pictures are shown in Fig. 1. The correlation coefficient values $(r$ ) between the two factors are presented in Table 2.

Increase in levels of $\operatorname{IgG}$, IgG1, IgG2, IgA and IgE anti-Leishmania in $\mathrm{SD}$ and $\mathrm{CD}$ groups was observed compared to control group (ND). As for IgM, there was no change in the response profile among the evaluated groups. When evaluating the association between the profile of anti-Leishmania antibodies and the different clinical pictures, it was observed that serum levels of IgG2 $(r=0.12)$ and IgM $(r=0.38)$ showed positive correlation with the clinical signs (Table 2). It was also observed negative correlation between serum levels of total $\operatorname{IgG}(r=-0.28), \operatorname{IgG} 1 \quad(r=-0.13)$

Table 1

Clinical signs observed in dogs, in group CD, naturally infected by Leishmania chagasi.

\begin{tabular}{lll}
\hline Clinical sign & $n$ & $\%$ \\
\hline Onychogryphosis & 23 & 33.82 \\
Hepatosplenomegaly & 31 & 45.59 \\
Cachexia & 53 & 77.94 \\
Lymphadenopathy & 38 & 55.88 \\
Keratoconjunctivitis & 42 & 61.76 \\
Skin ulcers & 24 & 35.29 \\
Apathy & 19 & 27.94 \\
Alopecia & 21 & 30.88 \\
More than one clinical sign & 59 & 86.76 \\
\hline
\end{tabular}




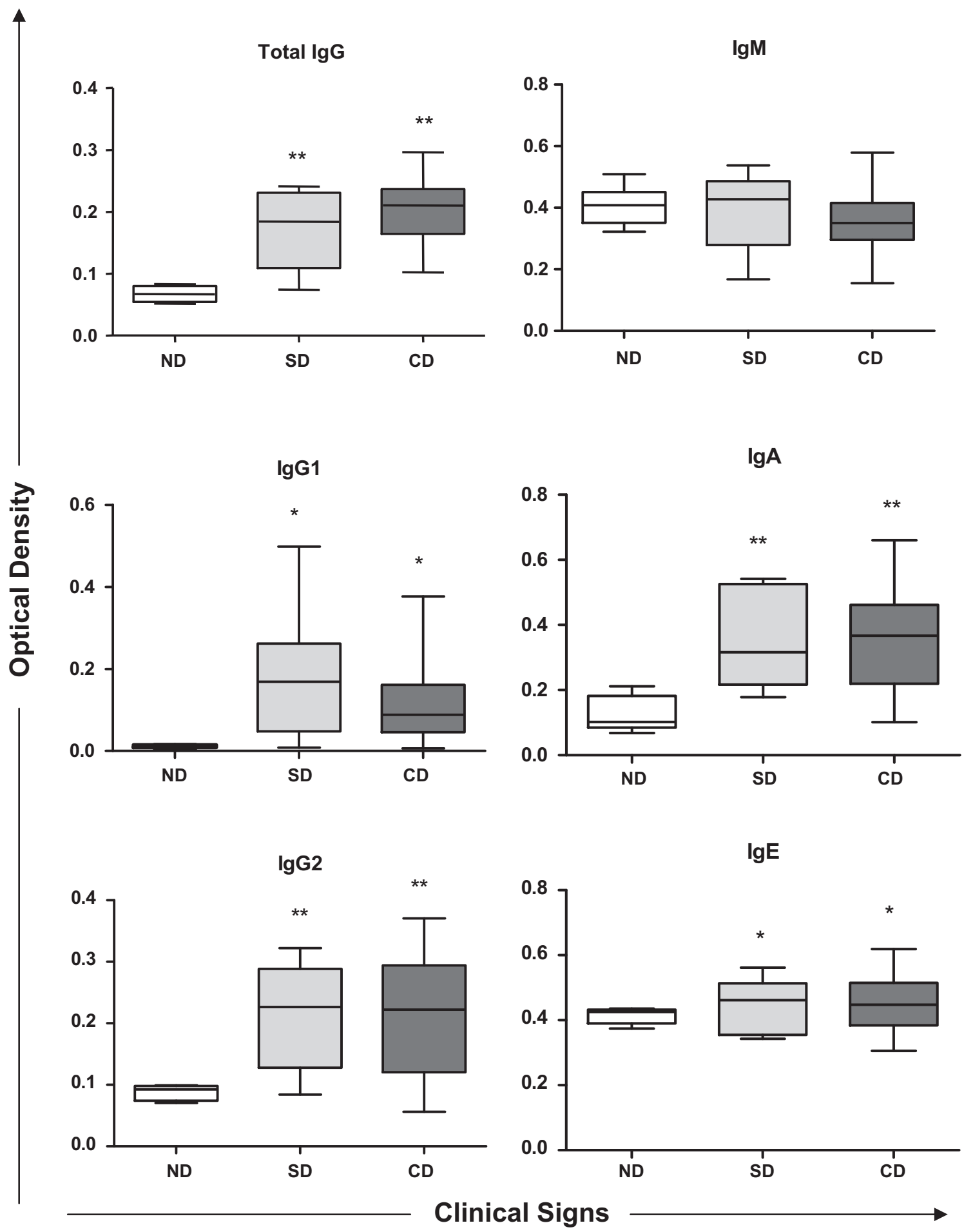

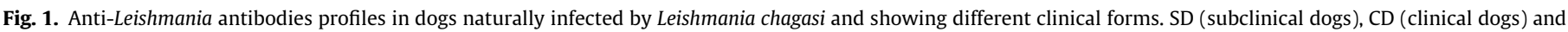
ND (negative dogs). ${ }^{*}$ Represents difference with a significance level of $5 \%$ and ${ }^{* *}$ represents difference with a significance level of $1 \%$.

and IgA $(r=-0.62)$ and the clinical signs. Moreover, IgE levels showed no correlation between SD and CD $(r=0.0)$, i.e., in these animals the increases of serum IgE are not correlated with the clinical changes evident in infected animals.

\section{Discussion}

It has been reported that the appearance of clinical signs characteristic of visceral leishmaniasis is mainly as consequences of the host immune response associated with deposition of immune complexes soluble in different tissues (Quinnell et al., 2003). In this direction, it could be interesting to assess the serum levels of anti-Leishmania antibodies verifying the classes and subclasses of immunoglobulins involved, and their association with clinical signs shown by infected animal.

In our study, we found that dogs naturally infected by Leishmania chagasi (CD and $\mathrm{SD}$ ) showed results of total IgG, IgG1, IgG2, IgA and IgE anti-Leishmania significantly increased when compared to ND (Fig. 1). Furthermore, we observed a negative 
Table 2

$P$ and $r$ values of anti-Leishmania antibodies profiles. Where $P$ compares differences in absorbance of immunoglobulins in different clinical forms (SD and $\mathrm{CD}$ ) with the negative control group (ND) of naturally infected dogs by Leishmania chagasi. $r$ value determines the correlation coefficient between immunoglobulins profiles and the clinical signs (CD) of naturally infected dogs by Leishmania chagasi.

\begin{tabular}{llllr}
\hline Immunoglobulin & \multicolumn{2}{l}{} & $r$ value & \\
\cline { 2 - 3 } & $\mathrm{ND} \times \mathrm{SD}$ & $\mathrm{ND} \times \mathrm{CD}$ & $\mathrm{SD} \times \mathrm{CD}$ & \\
\hline Total IgG & 0.009 & 0.007 & 0.12 & -0.28 \\
IgG1 & 0.02 & 0.04 & 0.08 & -0.13 \\
IgG2 & 0.009 & 0.008 & 0.11 & 0.12 \\
IgM & 0.08 & 0.11 & 0.09 & 0.38 \\
IgA & 0.009 & 0.008 & 0.09 & -0.62 \\
IgE & 0.04 & 0.03 & 0.07 & 0.00 \\
\hline
\end{tabular}

correlation between total $\operatorname{IgG}, \operatorname{IgG} 1$ and $\operatorname{IgA}$ and symptomatology $(r=-0.28, r=-0.13$ and $r=-0.62$, respectively), demonstrating that, the increase in these immunoglobulins is not associated with clinical signs of visceral leishmaniasis (Table 2).

Almeida et al. (2005) found that the titers of IgG anti-Leishmania in symptomatic dogs increased significantly when compared to asymptomatic and the control group. This fact was also found by Vercammen et al. (2002) that linked the increased levels of total IgG with progression from onset of symptoms.

In our study, the values of IgG1 anti-Leishmania showed a negative correlation coefficient with the clinical condition of the animals $(r=-0.13)$, and also their average serum levels decreased in animals showing clinical signs. These data corroborate the findings of Vercammen et al. (2002) and Cordeiro da Silva et al. (2003). With respect to IgG2 subclass, it happened differently, because the average serum absorbance values are almost the same as in all infected animals, not differing between CD and SD. However, these values are correlated with typical clinical signs of visceral leishmaniasis $(r=0.12)$, a fact which is mainly due to the findings of the $C D$ group.

It has been reported that the clinical signs are directly related to IgG1/IgG2 relationship, where the titers of the subclass IgG2 are strongly correlated with the symptoms of the animals. In this case, the titers of the subclass IgG1 also show increased, however with lower values than those found in IgG2 (Solano-Gallego et al., 2001; Leandro et al., 2001; Almeida et al., 2005).

Reis et al. (2006) found that IgG1 anti-Leishmania has a negative correlation with the clinical status of animals with visceral leishmaniasis, with their values diminished with clinical outcome, demonstrating association with the maintenance of the chronic and asymptomatic disease. With respect to $\operatorname{IgG} 2$, the same authors found that the values did not differ significantly between the infected animals, and only differed from the uninfected control group (Reis et al., 2006). These findings are in agreement with those obtained by other authors previously (Vercammen et al., 2002; Cordeiro da Silva et al., 2003).

In the present study, serum levels of IgM, anti-Leishmania from naturally infected dogs (SD and $C D$ ), remained with no significant differences compared to the control group (ND). However, with respect to the association with the symptomatology, there was a positive correlation coefficient between the groups and the clinical picture $(r=0.38)$. Yet, according to the one proposed by Reis et al. (2006), serum levels of IgM, in the L. chagasi infection, remain high until the chronic phase of disease. It is noteworthy, that this immunoglobulin is strongly associated with acute forms of parasitic diseases and that it does not present significant correlation with the clinical picture of CVL. Despite the above, little is known about the profile of IgM in CVL, because it is easily inactivated by reagents used in the main techniques of serology (Schallig et al., 2002), making it difficult to detect more accurate findings.

In relation to IgA anti-Leishmania, in our study, infected dogs showed negative correlation with the onset of the clinical picture of CVL $(r=-0.62)$, despite serum IgA levels were significantly increased $(P=0.0025)$ when compared to ND. Few studies emphasize the importance of IgA in the CVL. However, Reis et al. (2006) demonstrated a positive correlation of patterns of immunoglobulin-A with the clinical status of naturally infected animals. However, these data differ from our results. Whilst there are few data in literature, Day (2007) in his review reported that the levels of IgA anti-Leishmania were increased in the mucosa of infected dogs showing symptomatology, suggesting a source of aggravation of the infectious process.

It is noteworthy that dogs infected by Leishmania chagasi develop immune response mediated by Th 1 lymphocytes, they may be capable of preventing the spread of the parasite to the mucosal surface and, consequently, produce lower levels of specific IgA, with no differentiation with the clinical progression of CVL (Rodriguez-Cortes et al., 2007). Glomerulonephritis is a main clinical finding in the CVL, and the main triggering event of this abnormality has been attributed to the accumulation and deposition of immune complexes mediated by IgA in renal glomeruli (Nieto et al., 1992). Moreover, in post-mortem analysis, it was found that this clinical disorder is the leading cause of death of infected animals (Feitosa et al., 2000).

Regarding the levels of IgE anti-Leishmania, the results differed significantly between the infected animals (CD and SD) compared to non-infected $(P=0.0103)$. Still, when we evaluated the profile of IgE in the ND group, it was observed high absorbance values in some animals, which may represent false-negative results, by the diagnostic technique used, with possible evolution to a subclinical canine disease in these animals. These observations were also suggested by Almeida et al. (2005), who associated the IgE levels with the development of symptomatic cases of CVL. In this case, the predominant immune response is mediated by Th2 lymphocytes, which induce a decrease in the synthesis of IFN- $\gamma$, with increased expression of IL- 4 and IL-10, with data obtained by measuring the levels of mRNA (Quinnell et al., 2001).

In our study there was no correlation between the titers of IgE anti-Leishmania with symptomatology $(r=0.00)$, similar titers were detected in groups SD and CD. From these data we can consider that the appearance of symptoms is due to action of other immunoglobulins, and other elements of the immune response that were not objects of study. However works carried out by Iniesta et al. (2005) and Reis et al. (2006) observed a strong correlation between the titers of IgE and the clinical status of animals.

\section{Conclusion}

Our data demonstrate that dogs naturally infected with Leishmania chagasi present high titers of IgE, IgA and IgG2 anti-Leishmania and indicate that serum levels of IgG2 anti-Leishmania are correlated with typical clinical signs of disease, but serum levels of IgE antiLeishmania do not correlate with the disease progression. Further studies are needed to finally demonstrate that the determination of specific anti-Leishmania antibodies are an important tool to predict the clinical course of the disease as suggested by this study.

\section{Acknowledgments}

The authors would like to express their appreciation to the Fundação Cearense de Apoio ao Desenvolvimento Científico e Tecnológico (FUNCAP) for the financial help granted to the first author, which provided subsidies for the implementation of the project.

\section{References}

Almeida, M.A.O., Jesus, E.E.V., Sousa-Atta, M.L.B., Alves, L.C., Berne, M.E.A., Atta, A.M., 2005. Antileishmanial antibody profile in dogs naturally infected with 
Leishmania chagasi. Veterinary Immunology and Immunopathology 106, 151158.

Belkaid, Y., Tarbell, K., 2009. Regulatory T cells in the control of host-microorganism interactions. Annual Reviews of Immunology 27, 551-589.

Brasil, M.S., 2006. Manual de Vigilância e Controle da Leishmaniose Visceral, first ed. Editora MS, Brasília, pp. 11-18.

Ciaramella, P., Corona, M., 2003. Canine leishmaniasis: clinical and diagnostic aspects. VetLearn 25, 358-368.

Cordeiro da Silva, A., Cardoso, L., Araújo, N., Castro, H., Tomas, A., Rodrigues, M., Cabral, M., Vergnes, B., Sereno, D., Ouaissi, A., 2003. Identification of antibodies to Leishmania silent information regulatory 2 (SIR2) protein homologue during canine natural infections: pathological implications. Immunology Letters 86, $155-162$.

Day, M.J., 2007. Immunoglobulin G subclass distribution in canine leishmaniasis: a review and analysis of pitfalls in interpretation. Veterinary Parasitology 147,2-8

Feitosa, M.M., Ikeda, F.A., Luvizotto, M.C., Perri, S.H.V., 2000. Aspectos clínicos de cães com leishmaniose visceral no município de Araçatuba - São Paulo (Brasil). Clínica Veterinária 28, 36-44.

Freitas, J.C.C., Nunes-Pinheiro, D.C.S., Abreu, C.R.A., 2010. Geographical variation in clinical signs and prevalence of Leishmania sp. infection among dogs in Fortaleza, Ceará State, Brazil. Acta Scientiae Veterinariae 38, 293-297.

Ikeda, F.A., Luvizotto, M.C.R., Goçalves, M.E., Feitosa, M.M., Ciarlini, P.C., Lima, V.M.F. 2003. Perfil hematológico de cães naturalmente infectados por Leishmania chagasi no município de Araçatuba, São Paulo (Brasil): um estudo retrospectivo de 191 casos. Clínica Veterinária 47, 42-48.

Iniesta, L., Gallego, M., Portus, M., 2005. Immunoglobulin G and E responses in various stages of canine leishmaniosis. Veterinary Immunology and Immunopathology 103, 77-81.

Leandro, C., Santos-Gomes, G.M., Campino, L., Romão, P., Cortes, S., Rolao, N., Gomes-Pereira, S., Rica Capela, M.J., Abranches, P., 2001. Cell mediated immunity and specific $\operatorname{IgG} 1$ and $\operatorname{IgG} 2$ antibody response in natural and experimental canine leishmaniosis. Veterinary Immunology and Immunopathology 79, 273-284.

Miranda, S., Martorell, S., Costa, M., Ferrer, L., Ramis, A., 2007. Characterization of circulating lymphocyte subpopulation in canine leishmaniasis throughout treatment with antimonials and allopurinol. Veterinary Parasitology 144, 251-260.

Miyara, M., Sakaguchi, S., 2007. Natural regulatory T cells: mechanisms of suppression. Trends in Molecular Medicine 13, 108-116.

Nieto, C.G., Navarrete, I., Habela, M.A., Serrano, F., Redondo, E., 1992. Pathological changes in kidneys of dogs with natural Leishmania infections. Veterinary Parasitology 45, 33-47.
Peters, N., Sacks, D., 2006. Immune privilege in sites of chronic infection: Leishmania and regulatory T cells. Immunological Reviews 213, 159-179.

Quinnell, R.J., Courtenay, O., Shaw, M.A., Day, M.J., Garcez, L.M., Dye, C., Kaye, P.M., 2001. Tissue cytokine responses in canine visceral leishmaniasis. Journal of Infectious Disease 183, 1421-1424.

Quinnell, R.J., Courtenay, O., Garcez, L.M., Kaye, P.M., Shaw, M.A., Dye, C., Day, M.J., 2003. IgG subclass responses in a longitudinal study of canine visceral leishmaniasis. Veterinary Immunology and Immunopathology 91, 161-168.

Reis, A.B., Teixeira-Carvalho, A., Vale, A.M., Marques, M.J., Giunchetti, R.C., Mayrink, W., Guerra, L.L., Andrade, R.A., Corrêa-Oliveira, R., Martins-Filho, O.A., 2006. Isotype patterns of immunoglobulins: hallmarks for clinical status and tissue parasite density in brazilian dogs naturally infected by Leishmania (Leishmania) chagasi. Veterinary Immunology and Immunopathology 112, 102-116.

Ribeiro, V.M., 2007. Leishmaniose visceral canina: aspectos de tratamento e controle. Clínica Veterinária 71, 66-76.

Rodriguez-Cortes, A., Fernandez-Bellon, H., Ramis, A., Ferrer, L., Alberola, J., SolanoGallego, L., 2007. Leishmania-specific isotype levels and their relationship with specific cell-mediated immunity parameters in canine leishmaniasis. Veterinary Immunology and Immunopathology 116, 190-198.

Schallig, H.D.F.H., Schoone, G.J., Beijer, E.G.M., Kroon, C.C.M., Ozbel, Y., Ozensoy, S., Da Silva, E.S., Cardoso, L.M., Da Silva, E.D., 2002. Development of a fast agglutination screening test (FAST) for detection of anti-Leishmania antibodies in dogs. Veterinary Parasitology 109, 1-8.

Solano-Gallego, L., Riera, C., Roura, X., Iniesta, L., Gallego, M., Valladares, J.E., Fisa, R., Castillejo, S., Alberola, J., Ferrer, L., Arboix, M., Portus, M., 2001. Leishmania infantum-specific IgG, IgG1 and IgG2 antibody responses and healthy and ill dogs from endemic areas. Evolution in the course of infection and after treatment. Veterinary Parasitology 96, 265-276.

Solano-Gallego, L., Koutinas, A., Miró, G., Cardoso, L., Pennisi, M.G., Ferrer, L., Bourdeau, P., Oliva, G., Baneth, G., 2009. Directions for diagnosis, clinical staging, treatment and prevention of canine leishmaniosis. Veterinary Parasitology 165, 1-18.

Trotz-Williams, L., Gradoni, L., 2003. Disease risks for the travelling pet Leishmaniasis. In Practice 25, 190-197.

Vercammen, F., Fernandez-Perez, F.J., Del Amo, C., Alunda, J.M., 2002. Follow-up of Leishmania infantum naturally infected dogs treated with allupurinol: immunofluorescence antibody test, ELISA and Western-blot. Acta Tropica 84, 175-181. 